\title{
Numerical investigation on the liquid-liquid, two phase flow in a Y-shaped microchannel
}

\author{
X. Wang ${ }^{1}$ H. Hirano ${ }^{2} \quad$ N. Okamoto ${ }^{3}$
}

(Received 7 August 2006; revised 13 February 2008)

\begin{abstract}
A liquid-liquid, two phase, flow in a Y-shaped microchannel was numerically studied. Liquids 1 and 2, which are immiscible into each other, were injected into a Y-shaped microchannel from the two lateral channels respectively. The widths of the lateral and main channels are ten microns. The lengths of the lateral channels and main channel are five and 15 times of the channel width. The Volume of Fluid method was used to track the liquid-liquid interface, and PiecewiseLiner Interface Construction resolved a sharp interface. The interfacial tension was simulated with the Continuum Surface Force model with a wall adhesion boundary condition. The results show that a zebra flow mode appears in the main channel. For a certain inlet velocity, the length of zebra stripe depends on the interfacial tension force and inclined angles of two lateral channels. The length of a zebra stripe is
\end{abstract}

See http://anziamj.austms.org.au/ojs/index.php/ANZIAMJ/article/view/75 for this article, (c) Austral. Mathematical Soc. 2008. Published March 22, 2008. ISSN $1446-8735$ 
short at large interfacial tension. As for the effect of confluent angles of Y-shaped junction, the zebra stripe length is largest when the inclined angles of two lateral channels are $45^{\circ}$ and smallest when a T-shaped junction is used.

\section{Contents}

1 Introduction

C964

2 Model system

C966

3 Computational methods and governing equations

C967

3.1 Dimensional governing equation . . . . . . . . . . . . C967

3.2 Interfacial tension CSF model . . . . . . . . . . . . C968

3.3 PLIC reconstruction of the interface . . . . . . . . . C968

3.4 Non-dimensional governing equation . . . . . . . . . . C969

4 Result and discussion

C970

5 Conclusion

C973

References

C974

\section{Introduction}

Microfluidic technology is widely used in various fields due to the excellent mass and heat transfer properties as well as the uniform flow patterns and residence time distributions. Especially for a microreactor, some of these devices are available commercially and being tested by a growing community of researchers [1]. Recently, immiscible liquid-liquid segment (slug) flows in microchannels receive extensive exploration due to the segmentation of the 
two fluids, in which the chemical reaction, mixing and diffusion of the solutes are enhanced greatly by the increase of the interfacial area. On the other hand, microdroplets are produced in such a liquid-liquid system in the microchannel under certain conditions, for example, high inlet velocity of one phase, large interfacial tension and small contact angle between the fluid and the solid wall. The efficiency and properties of the segment (slug) flows in microreactors are mainly influenced by the channel geometry, viscosity ratio between two fluids, interfacial tension and the flux of the two liquids [2]. The variation in parameters imply that optimizing the segment (slug) flow system in microreactors needs extensive experimental work. Therefore, numerical study on such problems is indispensable to provide a reasonable and economical designing process.

For the numerical computation, accurate simulation of a multiphase flow problems with a moving interface lies in the obtaining of a sharp interface. Several techniques were developed in the last 25 years. Tang et al. [3] review these methods in detail. Rider and Kothe [4] discussed the historical perspective and comparison of various interface reconstruction techniques. In addition, in a multiphase flow, interfacial tension force plays a crucial role. A popular model for the interfacial tension force is the continuum surface force (CSF) model proposed by Brackbill et al. [5].

In the present study, a liquid-liquid two-phase flow in a Y-shaped microchannel was numerically studied due to its importance in the microreactor system, using VOF method with PLIC interface reconstruction technique, in which a cell-centered line segment was used to simulate the interface and the flux of fluid was computed geometrically according to the interface location and slope. The CSF interfacial tension model was adopted here to simulate the interfacial tension force. 


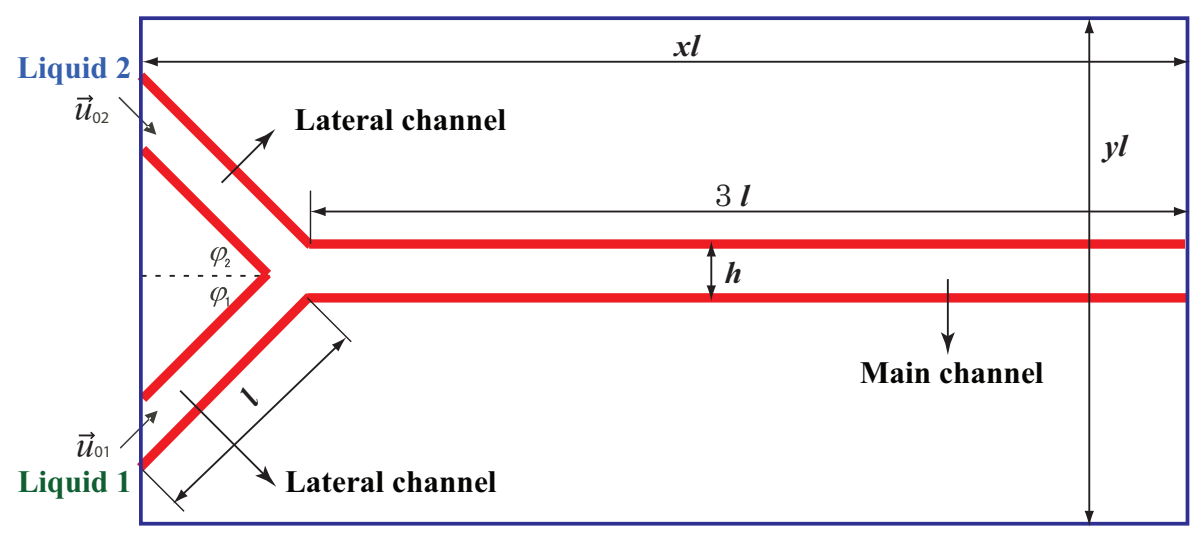

Figure 1: Computational model system.

\section{Model system}

Figure 1 shows the present model system. Liquids 1 and 2 were simultaneously injected into the Y-shaped microchannel from the two lateral channels with the same inlet velocities $\vec{u}_{01}=\vec{u}_{02}$. Both phases were assumed to be incompressible, Newtonian and immiscible at $1 \mathrm{~atm}$ and $25^{\circ} \mathrm{C}$. The flow was treated as laminar, which proved correct for the microflow under current situations [6]. Gravitational force was not taken into consideration due to its unimportance in the microflow. The widths $(h)$ of the lateral and main channels are same and equal to $10 \mu \mathrm{m}$. The lengths of the lateral channels and the main channel are $l=5 h$ and $3 l=15 h$, respectively. The inclined angles of two lateral channels are $\varphi_{1}$ and $\varphi_{2}$. A two dimensional computation was carried out and the domain $(x l \times y l)$ as shown in Figure 1. Here, $x l=l \cos \varphi_{1}+3 l$ and $y l=2 l+h .300 \times 220$ rectangular grids were adopted. 


\section{Computational methods and governing equations}

The interface of the two immiscible fluids was tracked by the volume of fluid (VOF) method [7] on Eulerian grids. The color function $C$ was set to 1 for liquid 1 and 0 for liquid 2. The interface was reconstructed by the Piecewise Liner Interface Calculation (PLIC) technique initially proposed by Youngs [8]. The surface tension was computed by the continuum surface force (CSF) model [5].

\subsection{Dimensional governing equation}

The dimensional governing equations follow. Color function:

$$
\frac{\partial C}{\partial t}+\frac{\partial(u C)}{\partial x}+\frac{\partial(v C)}{\partial y}=0
$$

Continuity equation:

$$
\frac{\partial u}{\partial x}+\frac{\partial v}{\partial y}=0
$$

Momentum equation:

$$
\begin{aligned}
& \frac{\partial u}{\partial t}+u \frac{\partial u}{\partial x}+v \frac{\partial u}{\partial y}=-\frac{1}{\rho} \frac{\partial p}{\partial x}+\frac{\mu}{\rho}\left(\frac{\partial^{2} u}{\partial x}+\frac{\partial^{2} u}{\partial y}\right)+\frac{1}{\rho} f_{\mathrm{svx}} \\
& \frac{\partial v}{\partial t}+u \frac{\partial v}{\partial x}+v \frac{\partial v}{\partial y}=-\frac{1}{\rho} \frac{\partial p}{\partial y}+\frac{\mu}{\rho}\left(\frac{\partial^{2} v}{\partial x}+\frac{\partial^{2} v}{\partial y}\right)+\frac{1}{\rho} f_{\mathrm{svy}} .
\end{aligned}
$$

Here, $\rho=\rho_{2}+C\left(\rho_{1}-\rho_{2}\right)$ and $\mu=\mu_{2}+C\left(\mu_{1}-\mu_{2}\right)$. Subscripts 1 and 2 denote for liquids 1 and 2 respectively. The flow field was solved with the finite difference method on a staggered mesh system. The HSMAC algorithm [9] was adopted to solve the pressure field and improve the velocity field. A non-slip velocity boundary condition was applied on the solid boundaries. 


\subsection{Interfacial tension CSF model}

$\vec{f}_{\mathrm{sv}}$ in equations (3) and (4) is the surface tension force per volume. In the CSF method, the surface tension is modeled as [5]

$$
\vec{f}_{\mathrm{sv}}=\sigma \kappa \frac{\nabla \rho}{\rho_{1}-\rho_{2}} \frac{\rho}{\left(\rho_{1}+\rho_{2}\right) / 2}=\sigma \kappa \frac{\rho}{\left(\rho_{1}+\rho_{2}\right) / 2} \nabla C .
$$

Here, $\sigma$ is the interfacial tension coefficient $[\mathrm{N} / \mathrm{m}] . \kappa$ is the local curvature of the interface curve $[1 / \mathrm{m}]$ and is calculated from

$$
\kappa=-\nabla \cdot \hat{n}=\frac{1}{|\vec{n}|}\left[\left(\frac{\vec{n}}{|\vec{n}|} \cdot \nabla\right)|\vec{n}|-(\nabla \cdot \vec{n})\right] .
$$

Here, $\hat{n}=\vec{n} /|\vec{n}|$ is the unit normal to the interface.

The effect of wall adhesion at fluid interfaces in contact with rigid boundaries is estimated easily in the CSF model by applying a boundary condition in equation (5):

$$
\hat{n}=\left(\cos \theta_{\text {ca }}\right) \hat{n}_{w}+\left(\sin \theta_{\text {ca }}\right) \hat{n}_{t},
$$

where $\theta_{\text {ca }}$ is the contact angle between fluid and solid wall. $\hat{n}_{w}$ is the unit wall normal and $\hat{n}_{t}$ is the unit tangent.

\subsection{PLIC reconstruction of the interface}

To get a sharp interface, the PLIC technique [8] was used to reconstruct the interface, in which a cell-centered line segment simulated the interface and the flux of fluid was computed geometrically according to the interface location and slope. The line segment is described as

$$
n_{x} x+n_{y} y+\eta=0 .
$$

According to the values of $C, n_{x}$ and $n_{y}$, the interface location and slope can be decided. Then, the flux of one phase whose color function is 1 is 
calculated analytically. In the present computation, the out-flow flux in a grid was computed, and the in-flow fluxes were corrected by the out-flow fluxes in the four neighbor grids. This is because the in-flow flux should be computed according to the values of $C$ in the neighbouring grid points.

\subsection{Non-dimensional governing equation}

Putting equation (5) into equations (3)-(4) and carrying out the dimensional analysis on the governing equations, we get the following non-dimensional governing equations. Color function:

$$
\frac{\partial C}{\partial \tau}+\frac{\partial(U C)}{\partial X}+\frac{\partial(V C)}{\partial Y}=0 .
$$

Continuity equation:

$$
\frac{\partial U}{\partial X}+\frac{\partial V}{\partial Y}=0 .
$$

Momentum equation:

$$
\begin{aligned}
& \frac{\partial U}{\partial \tau}+U \frac{\partial U}{\partial X}+V \frac{\partial U}{\partial Y}=-\frac{1}{\bar{\rho}+C(1-\bar{\rho})} \frac{\partial P}{\partial X} \\
& +\frac{\bar{\mu}+C(1-\bar{\mu})}{\bar{\rho}+C(1-\bar{\rho})}\left(\frac{\partial^{2} U}{\partial X}+\frac{\partial^{2} U}{\partial Y}\right)+\frac{2 \Gamma}{1+\bar{\rho}}(-\nabla \cdot \hat{n}) \frac{\partial C}{\partial X} \\
& \frac{\partial V}{\partial \tau}+U \frac{\partial V}{\partial X}+V \frac{\partial V}{\partial Y}=-\frac{1}{\bar{\rho}+C(1-\bar{\rho})} \frac{\partial P}{\partial Y} \\
& +\frac{\bar{\mu}+C(1-\bar{\mu})}{\bar{\rho}+C(1-\bar{\rho})}\left(\frac{\partial^{2} V}{\partial X}+\frac{\partial^{2} V}{\partial Y}\right)+\frac{2 \Gamma}{1+\bar{\rho}}(-\nabla \cdot \hat{n}) \frac{\partial C}{\partial Y} .
\end{aligned}
$$

The definition of the non-dimensional parameters are $X=x / x_{a}, Y=y / x_{a}$, $U=u / u_{a}, V=v / u_{a}, \tau=t / t_{a}, P=p / p_{a}, x_{a}=h, u_{a}=\mu_{1} / \rho_{1} x_{a}$, $t_{a}=\rho_{1} x_{a}^{2} / \mu_{1}, p_{a}=\mu_{1}^{2} /\left(\rho_{1} x_{a}^{2}\right), \bar{\rho}=\rho_{2} / \rho_{1}, \bar{\mu}=\mu_{2} / \mu_{1}, \Gamma=\sigma \rho_{1} x_{a} / \mu_{1}^{2}$. Here, subscript $a$ means the reference value. In the computations, $x_{a}=h=10 \mu \mathrm{m}$. Other conditions are $\rho_{1}=997.07 \mathrm{~kg} / \mathrm{m}^{3}, \mu_{1}=9.09 \times 10^{-4} \mathrm{Pas}, \bar{\rho}=1.2$, $\bar{\mu}=0.8$ and $\left|U_{01}\right|=\left|U_{02}\right|=0.1$. 


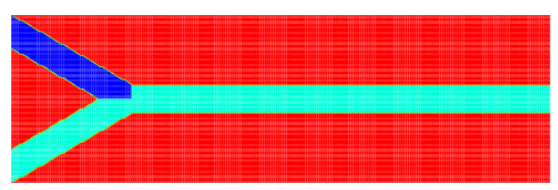

$\tau=0$

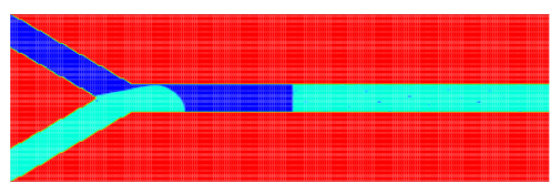

$\tau=40$

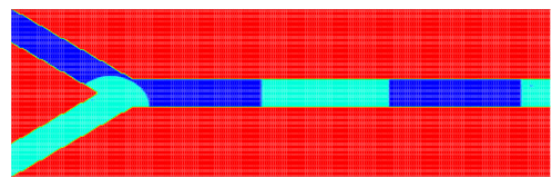

$\tau=80$

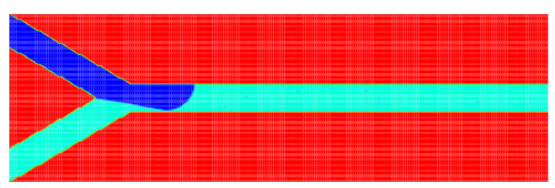

$\tau=20$

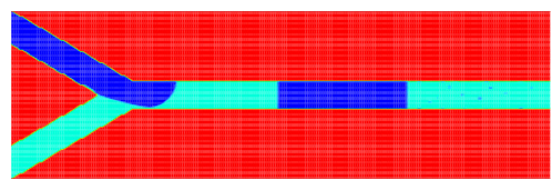

$$
\tau=60
$$

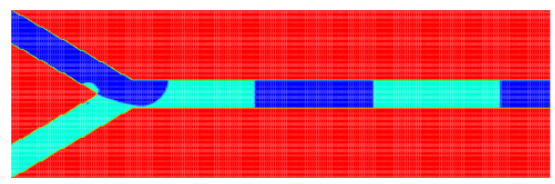

$\tau=100$

Figure 2: Instantaneous flow modes until $\tau=100$ at $\Gamma=250, \theta_{\text {ca }}=90^{\circ}$ and $\varphi_{1}=\varphi_{2}=30^{\circ}$.

\section{Result and discussion}

Figure 2 shows the instantaneous flow modes until $\tau=100$ at $\Gamma=250$, $\theta_{\text {ca }}=90^{\circ}$ and $\varphi_{1}=\varphi_{2}=30^{\circ}$. The initial condition for the phase field is shown with the picture of $\tau=0$. For the multiphase flow computation, the initial condition is very important: sometimes different initial condition cause different results. In the present work, the same initial condition were set for all the computational cases. At $\tau=20$, liquid 2 flows into the main channel. At $\tau=40$ the first slug of liquid 2 is produced near the crossing of the two lateral channels. At $\tau=60$, the second one starts to be generated and the same situation occurs as the first one at $\tau=20$. At $\tau=80$, the second slug of liquid 2 is produced. Such a process repeats in time. Segments of the two liquids are alternately generated and the zebra flow mode appears in the main channel. 


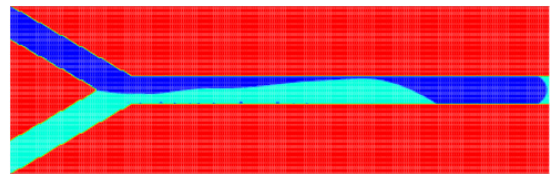

(a) $\theta_{\text {ca }}=30^{\circ}$

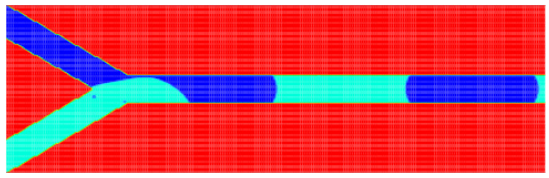

(b) $\theta_{\text {ca }}=60^{\circ}$

Figure 3: Flow modes under different contact angles at $\tau=80, \Gamma=250$ and $\varphi_{1}=\varphi_{2}=30^{\circ}$.

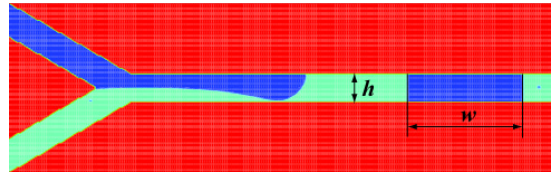

(a) $\Gamma=100$

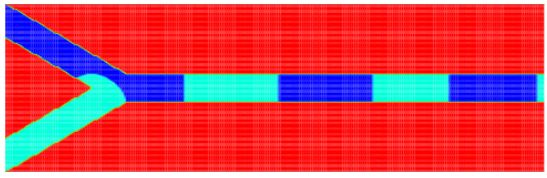

(b) $\Gamma=500$

Figure 4: Flow modes under different interfacial tensions $(\Gamma)$ at $\tau=80$, $\theta_{\text {ca }}=90^{\circ}$ and $\varphi_{1}=\varphi_{2}=30^{\circ}$.

Figure 3 shows the flow modes under different contact angles at $\tau=80$, $\Gamma=250$ and $\varphi_{1}=\varphi_{2}=30^{\circ}$. At same time moment $\tau=80$, almost one slug was produced at $\theta_{\text {ca }}=30^{\circ}$ (Figure $3(\mathrm{a})$ ), one and half at $\theta_{\text {ca }}=60^{\circ}$ (Figure $3(\mathrm{~b})$ ) and two at $\theta_{\text {ca }}=90^{\circ}$ (shown in Figure 2, $\tau=80$ ). On the other hand, at $\theta_{\text {ca }}=30^{\circ}$, the slug is produced far from the crossing of the two lateral channels. However, the contact angle does not affect the slug length.

Figure 4 shows the flow modes under different interfacial tensions at $\tau=$ $80, \theta_{\text {ca }}=90^{\circ}$ and $\varphi_{1}=\varphi_{2}=30^{\circ}$ : (a) is at $\Gamma=100$ and (b) at $\Gamma=500$. The nondimensional parameter $\Gamma$ indicates the ratio of interfacial force and viscous force on the fluid. The lengths of the zebra stripes are shorter at $\Gamma=500$ (Figure 4(b)) than those at $\Gamma=100$ (Figure 4(a)). On the other hand, at the same time moment $\tau=80$, the numbers of generated slugs are 1 , 2 and 3 for $\Gamma=100,250$ (shown in Figure 3, $\tau=80$ ) and 500, respectively. Thus the rate of production of slugs increases with the interfacial tension, which is very important for enhancing the performance of chemical reaction, 


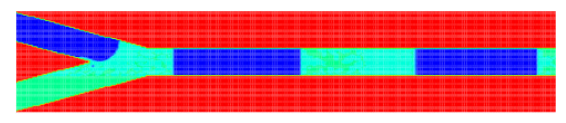

(a) $\varphi_{1}=\varphi_{2}=15^{\circ}$

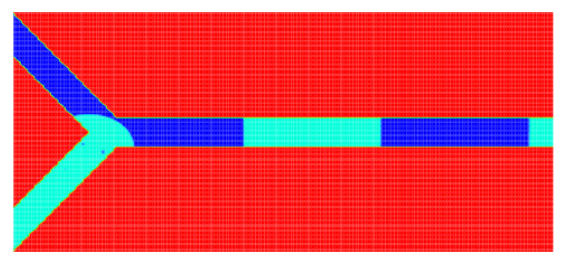

(b) $\varphi_{1}=\varphi_{2}=45^{\circ}$

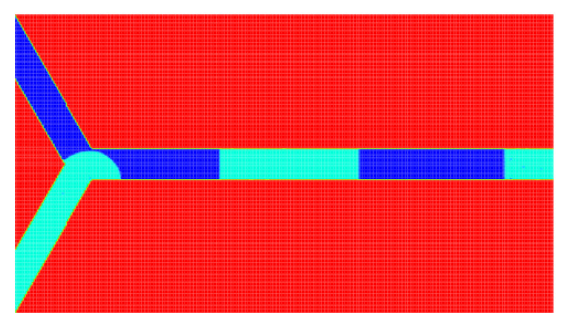

(c) $\varphi_{1}=\varphi_{2}=60^{\circ}$

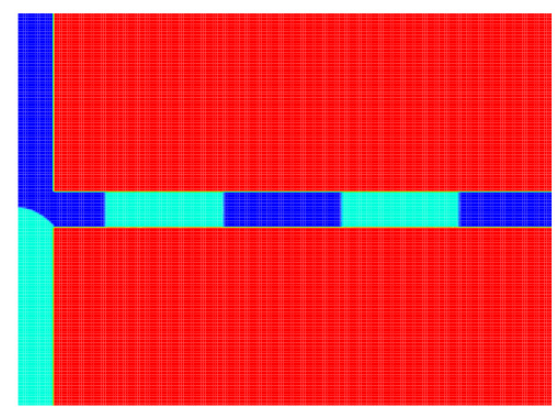

(d) $\varphi_{1}=\varphi_{2}=90^{\circ}$

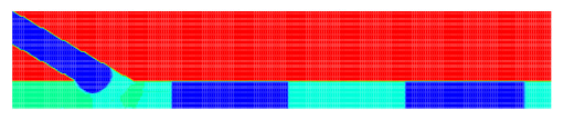

(e) $\varphi_{1}=0^{\circ}, \varphi_{2}=30^{\circ}$

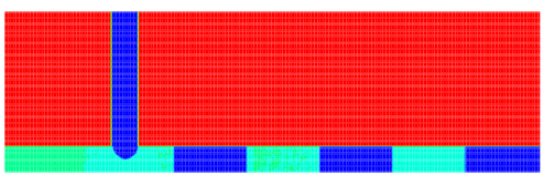

(f) $\varphi_{1}=0^{\circ}, \varphi_{2}=90^{\circ}$

FiguRE 5: Flow modes under various inclined angles of two lateral channels, $\varphi$, at $\tau=80, \theta_{\text {ca }}=90^{\circ}$ and $\Gamma=250$.

extraction, mixing and increasing the yields of micro particles/droplets in the microreactor system. Besides, the location where the slug is generated becomes further from the crossing of two lateral channels as $\Gamma$ decreases. At $\Gamma=100$ (Figure 4(a)), the slug is generated at the middle of the main channel and a parallel flow appears in the upstream of the channel. However, at $\Gamma=500$ (Figure 4(b)), the slug is generated just at the beginning of the main channel. Therefore, a long channel is needed for two kinds of liquids with small interfacial tension to generate zebra flow mode. With $\Gamma$ decreasing, the parallel flow mode occupies the channel more and more before a slug is generated. Especially, at $\Gamma=0$, instead of the zebra flow mode, the parallel flow appears in the whole channel and no slug is produced. 
TABLE 1: The value of $w / h$ under various inclined angles at $\Gamma=250$.

\begin{tabular}{|cc|cc|}
\hline$\varphi$ & $w / h$ & $\varphi$ & $w / h$ \\
\hline$\varphi_{1}=\varphi_{2}=15^{\circ}$ & 4.39 & $\varphi_{1}=\varphi_{2}=75^{\circ}$ & 3.39 \\
$\varphi_{1}=\varphi_{2}=30^{\circ}$ & 4.67 & $\varphi_{1}=\varphi_{2}=90^{\circ}$ & 3.14 \\
$\varphi_{1}=\varphi_{2}=45^{\circ}$ & 4.95 & $\varphi_{1}=0^{\circ}, \varphi_{2}=30^{\circ}$ & 4.17 \\
$\varphi_{1}=\varphi_{2}=60^{\circ}$ & 4.60 & $\varphi_{1}=0^{\circ}, \varphi_{2}=90^{\circ}$ & 2.71 \\
\hline
\end{tabular}

Figure 5 shows the flow modes under various inclined angles of two lateral channels at $\tau=80, \theta_{\text {ca }}=90^{\circ}$ and $\Gamma=250$. The length of zebra stripe strongly depends on the inclined angles $\left(\varphi_{1}\right.$ and $\left.\varphi_{2}\right)$. Table 1 shows the value of $w / h$ under various inclined angles. Here, $w$ is the length of one of the zebra stripes shown in Figure 4(a). Since the inlet velocities of the two phases are the same and the channel width (both lateral and main) is the same everywhere, the stripe lengths of two liquid are the same. Table 1 shows when $\varphi_{1}=\varphi_{2}$ and smaller than $45^{\circ}$, the value of $w / h$ increases with $\varphi$. At $\varphi_{1}=\varphi_{2}=45^{\circ}$, the value reaches its largest value 4.95 . When $\varphi_{1}=\varphi_{2}$ and larger than $45^{\circ}$, the value of $w / h$ decreases with the increase in $\varphi$. At $\varphi_{1}=\varphi_{2}=90^{\circ}, w / h$ is smallest and equals to 3.14. For all the cases shown in Table 1 , the smallest value of $w / h$ is at $\varphi_{1}=0^{\circ}$ and $\varphi_{2}=90^{\circ}$. Consequently, obtain more segment/interfaces of the two liquids using T-shaped junction $(\vdash$ or $\perp)$.

\section{Conclusion}

A liquid-liquid, two phase, flow in a Y-shaped microchannel was numerically studied. Using the PLIC technique, a sharp interface of two fluids was simulated. A zebra flow mode was found in the Y-shaped microchannel. At a certain inlet velocity, the length of the zebra stripe or the number of liquidliquid interfaces, which is very important for the mass transfer between two phases, is strongly dependent on the interfacial tension and the confluent 
angle of the Y-shaped junction. When the interfacial tension is large, the zebra stripe length is small and the parallel flow which appears before a slug is generated is short. As for the effect of inclined angles of lateral channels, the T-shaped junction $(\vdash$ or $\perp$ ) tends to produce a short zebra stripe flow mode and more interfaces between the two liquids.

Acknowledgements This work was supported by the project of Cooperation of Innovative Technology and Advanced Research in Evolutional Area (CITY AREA) by Okayama Prefecture Industrial Promotion Foundation from the Ministry of Education, Culture, Sports, Science and Technology, Japan.

\section{References}

[1] W. Ehrfeld, V. Hessel, and H. Lowe. Microreactors, New Technology for Modern Chemistry. WILEY-VCH Verlag GmbH, D-69469 Weinheim (Federal Republic of Germany), 2000. C964

[2] Harries, N., Burns, J. R., Barrow, D. A. and Ramshaw, C., A numerical model for segmented flow in a microreactor, International Journal of Heat and Mass Transfer, 46, 2003, 3313-3322. C965

[3] Tang, H., Wrobel, L. C. and Fan, Z., Tracking of immiscible interfaces in muitiple-material mixing processes, Computational Materials Science, 29, 2004, 103-118. C965

[4] Rider, W. J. and Kothe, D. B., Reconstrcting volume tracking, J. Comp. Phys. 141, 1998, 112-152. C965

[5] Brackbill, J. U., Kothe, D. B. and Zemach, C., A continuum method for modeling surface tension, J. Comp. Phys. 100, 1992, 335-354. C965, C967, C968 
[6] V. Hessel, S. Hardt and H. Lowe. Chemical micro process engineering, Fundamentals, modelling and reactions. WILEY-VCH Verlag GmbH \& Co.KGaA, Weinheim, 2004. C966

[7] Hirt, C. W. and Nichols, B. D., Volume of fluid (VOF) method for the dynamics of free boundaries, J. Comput. Phys. 39, 1981, 201-225. C967

[8] Youngs, D. L., Time-dependent multi-material flow with large fluid distortion, in: Numerical Methods for Fluid Dynamics, edited by K. W. Morton and M. J. Baines, Academic Press, New York, 1982, 273-285. C967, C968

[9] C. W. Hirt, B. D. Nichols and N. C. Romero. SOLA: A numerical solution algorithm for transient fluid flow-Addendum. Los Alamos Scientfic Laboratory Report, LA-5852, 1975. C967 


\section{Author addresses}

1. X. Wang, Dept. Applied Chemistry, Okayama University of Science, Okayama, JAPAN.

mailto:wangxian77@yahoo.com.cn

2. H. Hirano, Dept. Applied Chemistry, Okayama University of Science, Okayama, JAPAN.

mailto:hirano@dac.ous.ac.jp

3. N. Okamoto, Dept. Applied Chemistry, Okayama University of Science, Okayama, JAPAN. 